\title{
The Ideological Limits of Digital Poetics
}

\author{
Tirtha Prasad Mukhopadhyay \\ Professor and Research Fellow, University of Guanajuato, Mexico \\ Email: chiefeditor@rupkatha.com
}

\begin{abstract}
Digital poetics has emerged as a genre following the development of machine augmented literatures. An algorithm could generate transient but successful emotive appraisals that characterize human poetry. Production of cybertext is enabled by the presence of advanced Anglophone Synset compilations, text summarization and development-constrained specifics of the corpora. Digital poetics in regional nonAnglophone languages thus remain marginalized owing to language dependency across the world's media divide. Whereas such hierarchies are inevitable in a technologically divided world the recognition of these problems and logical solutions may help foment innovation, entrepreneurship and affirmative opportunities for underdeveloped economies.
\end{abstract}

Keywords: Digital Poetics, Cybertext, Hypertext, Indowordnet

\section{Coding: Certain Limitations}

In online literature literary hypertexts were first proverbially generated in an interactive fashion or with a considerable open-ended trajectory; each click on a link in the narrative lead to another page and further links, creating extended fictional spaces in a randomized manner. The hypertextual novel, whether in narrative or visual media, especially in such text-visual creations as Paul Laforge's and Alcorn's The Art of the Missing would be a fairly open-ended generative narrative: yet the links are confined to beliefs cordoned off by the limits of a certain language and its cognitive spaces. These limits are cultural, religious long-held beliefs. Katherine Hayles formulated an appreciation or 'theory' of such texts that came to describe a hypertext critique tailored mostly for the Anglophone American academy, with its subconscious socio-industrial enbeddedness. Hayles appeals only to the superficial, fragmented and dialogic structure of hypertext narrative. She tries to show that "electronic literature is not print", which implies that electronic texts are categorically different from conventional stories and are sensitive to coding. Hayles refers to Lev Manovich's important distinction from the Language of the New Media where Manovich says that that electronic literature is a literature that is essentially coded (Manovich 2002) - that its structure and meaning in time are determined by the open codex form of access to the text and the readerly capacities of engendering meaning. Hayles suggests that

"Unlike a print book, electronic text literally cannot be accessed without running the code. Critics and scholars of digital art and literature should therefore properly consider the 
source code to be part of the work, a position underscored by authors who embed in the code information or interpretive comments crucial to understanding the work." (Hayles 2007. My italics)

In other words, the thrust of the argument lies in the underlayering concept of the code. Beyond Hayles and much into the literature of generative consciousness, again as Hayles herself pointed out, the importance of coding is even greater for an artificially intelligent coding machine which might write narratives, and with codes which one day would probably enable machines to generate fictions and novels. The distinction between digital literature, that is conventional narrative and digitized, and AI Augmented digital literature does not appear significant when we realize that in both cases the narrative is produced either (a) open-source coding or (b) pretraining. But even where Hayles, following Malevich, emphasizes on coding what remains to be said from the perspective of hardware limitations is that the scale of neural (human) coding makes the dream of a completely machine-generated humanist text unthinkable and impossible to achieve, even by today's standards of hardware technology (Goser 1996). There are no human resources, neither of intelligence or comprehension, nor artificial computing that could make a self-intelligent machine operative in the same way as a human writer functioned. Limitations imposed by the mind-body behavioral duality will not allow re-structuring textual synthesis to the extent that the human narrator - and by extension - a humane author - could possibly write a text, and accordingly a human reader could engage with, in any meaningful way.

\section{Ideological Limitations}

What this means, we argue, is that even coding has to automatically incorporate an ideology that is conditioned by relational semantics that is always intrinsic to any proto-human system of language. We could show what the differences and similarities among various textual media imply about the theories and practices of machine generated literature. Aspen Aarseth claimed for the possibility of NLG-based literary and aesthetic textuality on the digital media - and notably introduced concept of the Cybertext.

"Cybertext is a perspective on all forms of textuality, a way to expand the scope of literary studies to include phenomena that today are perceived as outside of, or marginalized by, the field of literature-- or even in opposition to it." (Aarseth 1997)

Let us consider here the kind of narrative developed by such AI augmented programs as the GPT3 model. Recently, the GPT-3 model has drawn a lot of attention: "AI research and deployment company OpenAI - founded by Elon Musk, Sam Altman, and others - announced its revolutionary language model, GPT-3." (https://openai.com/blog/openai-api/ retrieved 9th March 2021). David Chalmers recommends GPT-3 AI modularity as one of the most semantically efficacious examples of machine literature. Yet, Amanda Askell raises an important question in this regard. Askell calls the AI literature model of the GPT-3 and other similar ones as 'Renaissance Models'. Askell asks

"GPT-3 recently captured the imagination of many technologists, who are excited about the practical applications of a system that generates human-like text in various domains... But GPT-3 also raises some interesting philosophical questions. What are the limits of this approach to language modeling? What does it mean to say that these models (i.e. machines) 
3 | The Ideological Limits of Digital Poetics

generalize or understand? How should we evaluate the capabilities of large language models?" (Askell 2019)

Even Aarseth's functional approach to texts, like in Hayles' position, leaves questions of ideology and allegiance aside. Indeed, any propositional context that may contain a message, is a problematic operation for any kind of conventional textuality just as for texts generated out of machine intelligence: there are no ideologically neutral texts (De Schutter 2008). Texts are always habitus valorizing because they involve rhetorical strategies that are biopolitically linked and extended out of the habitus of its experience (Scannell 2007).

"These findings suggest a complex moral, social, and political problem space, rather than a purely technological one. Not all uses of AI, of course, are inherently objectionable, or automatically unjust - the point is simply that much like we can do things with words, we can do things with algorithms and machine learning models. This is not purely a tangibly material distributive justice concern: especially in the context of language models like GPT3 , paying attention to other facets of injustice-relational, communicative, representational, ontological-is essential." (Scannell 2007)

\section{What is Pre-training? Any Hope in It?}

The problem of contending with inbuilt ideologies appears during pre-training of programmable contexts of text generation. What is pretraining? "Pre-training in AI refers to training a model with one task to help it form parameters that can be used in other tasks. The concept of pre-training is inspired by human beings. ... that is: using model parameters of tasks that have been learned before to initialize the model parameters of new tasks" (https://medium.com retrieved 20 ${ }^{\text {th }}$ November 2021). What does the limits set to pre-training imply for content-coding of electronic literatures? It is safer for us to understand the problem by retracing the creative pathway as espoused by Aarseth. Aarseth does not really see any distinction between electronic and print narrative, in the way Hayles supposes this dichotomy to exist. But for AI literature pre-training will remain the fundamental crux around which we could peg the debates of the future. Programming is the bottleneck through which ideologically sensitive pre-training shall not appear to pass. Pretraining, as we know, is the way an AI machine will assess or re-assess its database based on the feedback of interactions, and then self-modify itself to adapt to communicative strategies. But pretraining data bases will of course be value laden, just as block chains are value laden for corporal (identity) fingerprints since semantics will bind the nature of human relationships and hierarchies in a class based human world.

If we accept Aarseth's "ergodic" formula for the most flexible systems of tagging-management we see how this already starts creating restrictions on the level of subroutine programming. Even in the latest operating systems which allow for enormous flexibility in the adaptation of parameters as aspects of the subroutine - there are limitations of upper-level tagging argument, as most of the data sets are those not outside the spectrum of our common languages. This is a problem for AI. It is also a problem for electronic literature as it is available on the Web 2.0 and possibly Web 3.0 applications and software. The intelligent human author can have access to a greater number of tagging parameters in a very short instant of time and is able to improvise on 
the existence of the program itself. In this sense the computer can only act or serve as a pet dinosaur for the programmer - one already extinct because computation restricts generative narratives by the syntactical determinism of its storage and retrieval. Pre-training excludes dimensional length and extent, as well as the brilliantly absurd specificity of human generated meaning. For digitized, that is archived platforms, which offer singular bibliographical access to conventional literature - or literature as we understand it - as well as transmedia and pixel collage which brings together multiple media elements into the narrative - in all these cases without exception - programming is hyperlinked as ludic transition for the reader in the same manner. In other words, hypertextuality is a human value module. It has enhanced possibilities of expression on the digital platform but is not really a self-determining feature for the political discourse of new media.

Cyborg aesthetics would, by definition, provide a material culture replacement of conventional 'pre-anthropocene' old world art formats - involving platform change and inclusion of multimedia or games to both untrivial and trivial creativity (Aarseth 1997). The hypercard application developed by Noah Wardrip-Fruin and David Durand, also reminds us of digital poetics in this regard and also of Loss Pequeño Glazier's Digital Poetics. Glazier has installed sense in the claim that electronic poetry is a continued expression of literate written poetry. Electronically archived poetry intrinsically carries the meaning of written poetry when it is retained in the scripted form, not though when the language itself changes. The Anglophone archive can launch an anglophone meaning chain which is both made universally available for reflection, criticism and re-creation, but again which also creates invasive parameters on the internet of things. Among various other elements I shall consider two dimensions of the habitus morphology that poetry acquires in its narratives, and thus celebrates. Poetics is more directly connected with brief verbal inputs that comprise tagging subroutines of a specific format. Fragments of poetic discourse lend themselves very swiftly to enjambment and networking through related verbal cues. Visual cues may be used as well: shape poems offer examples here. But as Glazier shows the final product would be a humanly understood message with human laden values in the social collective.

\section{Indowordnet. A Promise or Fall?}

What is Indowordnet? This data base, created as a tagging resource for a selection of languages from the Indian subcontinent paradoxically or unexpectedly compares Indian language words like that of Hindi with English semantic equivalents for Synset pairings in order to understand the exact ontological meaning of a word (Garg et al 2019). As is known for semantic databases word meanings of nominatives will have ontologies but the Indowordnet uses word references from Worldnet data base in English to define semantic taxonomies. In any programmable digital poetics a semantically unclassified ontology of words would prove to be a hindrance. Again, in Indowordnet the Synset which can provide the list of homonym-synonym correlations is less easily available. This is just to show how Synsets in languages other than English are not yet developed. Hence creation of artificially generated poetic texts gets restricted - indeed a vast amount of information on the nature of language itself would be revealed if the relationships between phonetic components or syllabary could be classified with meanings of words. Regional literatures are clearly at a disadvantage on the computer and so is creativity. The flexibility with which 
5 | The Ideological Limits of Digital Poetics

Raymond Queneau achieves his effects for his series of poems show the distinctive advantage that an English or French AI poet has over other AI generators. The case is more acutely visible in case of rendering translations. Indeed, a good translator of highly metaphorical language has not yet been developed - it will need years of fundamental research to create condensed poetic generators with metaphorical suggestivity, even though some progress has been made in relation to brief story narratives.

\section{Raymond Queneau's Digital Poetics}

We cannot ignore the importance of Raymond Queneau's poetry in this discussion (Queneau 2018). Networking metaphoric fragments is discussed by Umberto Eco's essay on Raymond Queneau and Barbara Wright's translation and foreword to Queneau, where Barbara Wright suggests that Queneau who experimented with sound rather than the grammatical foresight of the word, enables Queneau to join fragments of unrelated syllables to create a word. These lines from Queneau are an AI augmented parody of Shakespeare that uses the template to choose words and generate meaning for a specific moment or chance creation:

Like as the weapons make towards the pebbled shrapnel,

So do our misalliances hasten to their engine;

Each changing plaint with that which goes before,

In sequent tombs all forwards do contend.

(Praising Thy Wren retrieved 12th July 2021. https://mypoeticside.com/show-classicpoem-22973). (Queneau 2018)

The poem reveals the arbitrariness of the combination of lexical elements to create the same effects Shakespeare's ripple effects in the verse. The verbal energy of that verse, which in Shakespeare refers to the waves on the shore, is conditioned by the words that the computer uses to augment the template with very different lexical units and significance of meaning. Hence translation was not resisted, as long as that process could be carried forward from French to English or as Queneau does for his poem called Haiku, from Japanese to French, via a Tibetan misnomer corpus subset that guides that process. Eco called this artificially intelligent process of semantic formation a product of a natural metaplasm of meaning. The word 'metaplasm; at once challenges and deconstructs written or scripted poetic meaning, it is a self-reflected indicator of the meaning process. The metaplasm sticks and connects the word fragment out of its plenum of meaning - yet by itself it remains hidden to our eyes.

Yet unlike the advanced extent to which Digital Poetics become feasible in such projects as Queneau's Hundred Thousand Billion Poems (2020) or other projects like the Haiku (again in English) or tempslibres - Data Base of Haikus compiled since 2000, large data bases like the PoetRNN corpus or the Haikuzao corpus have facilitated such projects. Digital Poetics depends on maintenance of huge corpora, some of the most famous being the Gutenberg English poetry Corpus (GEPC) and the Shakespeare Online, http://www.shakespeareonline.com/sonnets/sonnetintroduction.html which are augmented and trained for poetry generation. Yet when corpus building in other non-global languages are compared to this, what 
appears is a vast gap in the scale of achievement. Digital Poetics have achieved little in other languages except French or German, because of a hegemonically operative neglect caused by human, social funding and entrepreneurial differences in these others when compared to economically advanced cultures. The fundamental problem that faces innovation crisis in the less developed, often Anglophone zones of Latin America, Asia and Africa is a cultural economic problem. The lack of sufficiently large and dedicated corpora, the lack of appropriate, textual summarization protocols and the lack of development of satisfactory constraint-based generators would reflect on the future of digital poetics in a way contrary to heterogeneity, multiculturality and regional identity and self-esteem.

\section{Conclusion}

Umberto Eco's concept of metaplasm is synonymous with the Indian grammarian Bhartrihari's notion of the pashyanti - the paradoxical word that Bhartrihari used to describe semantic generation. Bhartrihari refers to meaning as a product of the act of looking vis a vis at meaning it means looking at what is invisible about meaning, its falling into place so to speak. To 'see' here, by analogy, is to compute meaning out of the possibilities of discourse. Eco's metaplasm, following Queneau's poetics in the Western world, and Bhartrihari's pashyanti is essentially discretely computed in digital scripts, encrypted as it were by a code where script is protected and archived forever, and is yet also a testimonial process in so far as the poetics is enabled in terms of the training of a corpus to achieve permutational effects when a new sequence of lexemes are joined together. This metaplasm of Artificial Intelligence may be different from human intelligence. Based on the computational model semantic relationships in AI authored texts will be based and reified as semantic trees. If the human generated metaplasm has another kind of growth and expression, one of very deep hybridizing and recreation of semantic vestiges on different levels or branches of cognitively realized emotions then digital poetics may not be feasible beyond a certain extent, as least beyond the extent to which the machine can enable this in the foreseeable future (Chomsky 1976, 2000). However, acquired, artificially evolved poetic expression may also not be able to free itself, unless in a self-reflexive way, from the hierarchizing attributes of human existence, capital inequities, deprivations and unequal wealth affiliated valuations. The lack of AI augmented generators for poetry in underdeveloped regions of the world is likely to affect us in two ways. First there is the trailing lack of technological advancement leading to a lack of advances in machine analysis of human sentiments and sentiment generation. This component may be vital to machine human interactions with more emotive consequences, in the society of the future which will increasingly involve working with robots and generators. The other drawback for regional cultoromics may be apprehended in that they are likely to be left behind in innovations and this will continue to challenge existential perceptions of the future.

\section{References}

Aarseth, E J. (1997). Cybertext: Perspectives on Ergodic Literature. USA: The John Hopkins University Press.

Askell, Amanda, Miles Brundage, and Gillian Hadfield. (2019) "The role of cooperation in responsible AI development." arXiv preprint arXiv:1907.04534. 
7 | The Ideological Limits of Digital Poetics

Baxter, R. N. (2009). New technologies and terminological pressure in lesser-used languages: The Breton Wikipedia, from terminology consumer to potential terminology provider. Language Problems and Language Planning, 33(1), 60-80.

Bhatt, Brijesh, and Pushpak Bhattacharyya. (2011). "IndoWordNet and its linking with ontology." In Proceedings of the 9th International Conference on Natural Language Processing (ICON-2011)

Bourdieu, Pierre. (2020). Habitus and Field: General Sociology, Volume 2 (1982-1983). Polity Press.

Chomsky, Noam. (1976). "On the biological basis of language capacities." In The neuropsychology of language, pp. 1-24. Springer, Boston, MA

Chomsky, Noam. (2000). New horizons in the study of language and mind. Cambridge University Press

De Schutter, H. (2007). "Language policy and political philosophy: On the emerging linguistic justice debate." Language problems and language planning, 31(1), 1-23.

Drori, Gili S. (2010). "Globalization and technology divides: Bifurcation of policy between the "digital divide" and the "innovation divide"." Sociological Inquiry 80, no. 1 (2010): 63-91.

Garg, Amita, and Jatinderkumar Saini. (2019). "A Systematic and Exhaustive Review of Automatic Abstractive Text Summarization for Hindi Language."

Gee, J P. (2003). "What video games have to teach us about learning and literacy." Computers in Entertainment. October. 1:1. 20-20

Goser, Karl F. (1996). "Implementation of artificial neural networks into hardware: Concepts and limitations." Mathematics and computers in Simulation 41, no. 1-2 (1996): 161-171.

Hayles, N. Katherine. (2007). "Electronic Literature: What is it?." Doing Digital Humanities: Practice, Training, Research.

Manovich, Lev. (2002). The language of new media. MIT press

Michel, Jean-Baptiste et al. (2011). "Quantitative analysis of culture using millions of digitized books." science 331, no. 6014

Peacock, A., (2010). "Towards an aesthetic of 'the interactive." Digital Creativity. August. 12:4

Pimienta, Daniel et al. (2009). "Twelve years of measuring linguistic diversity in the Internet: balance and perspectives". United Nations Educational, Scientific and Cultural Organization.

Pinker, Steven. (2010). "The cognitive niche: Coevolution of intelligence, sociality, and language." Proceedings of the National Academy of Sciences 107, no. Supplement 2 (2010): 8993-8999.

Queneau, Raymond. (2018). Exercises in style. Alma Books

Scannell, K. P. (2007). "The Crúbadán Project: Corpus building for under-resourced languages." In Building and Exploring Web Corpora: Proceedings of the 3rd Web as Corpus Workshop (Vol. 4, pp. 5-15).

Shevlin, Henry et al. (2020). "If You Can Do Things with Words, You Can Do Things with Algorithms."

Song, J. J. (2011). English as an official language in South Korea: Global English or social malady?. Language Problems and Language Planning, 35(1), 35-55.

Thorne, S. L. (2008). " Bridging activities," new media literacies, and advanced foreign language proficiency. Calico Journal, 25(3), 558.

Van Dijk, Z. (2009). Wikipedia and lesser-resourced languages. Language Problems and Language Planning, 33(3), 234-250. 